\title{
Temperature, Daylength, and Cultivar Interact to Affect the Growth and Yield of Lettuce Grown in High Tunnels in Subtropical Regions
}

\author{
Ah-Chiou Lee \\ Department of Horticulture and Landscape Architecture, National Taiwan \\ University, No. 1, Sec. 4, Roosevelt Road, Taipei, Taiwan; and Taoyuan \\ District Agricultural Research and Extension Station, Council of Agriculture, \\ Executive Yuan, No. 139, Sec. 2, Dongfu Road, Hsinwu, Taoyuan County \\ 32745, Taiwan
}

\section{Fang-Shin Liao}

Taoyuan District Agricultural Research and Extension Station, Council of Agriculture, Executive Yuan, No. 139, Sec. 2, Dongfu Road, Hsinwu, Taoyuan County 32745, Taiwan

Hsiao-Feng Lo ${ }^{1}$

Department of Horticulture and Landscape Architecture, National Taiwan University, No. 1, Sec. 4, Roosevelt Road, Taipei, Taiwan

Additional index words. Lactuca sativa, landrace, growth rate, breeding, correlation, multiple regression

\begin{abstract}
The production of lettuce, a cool-season leafy vegetable, in high tunnels the year around is a challenge for growers in subtropical regions. The aims of this research were to characterize the growth of locally grown lettuce cultivars, develop a new high-yielding cultivar by crossing romaine-type lettuce 'Jhih $\mathrm{Li} \mathrm{Wo'} \mathrm{and} \mathrm{Batavia} \mathrm{type} \mathrm{lettuce} \mathrm{'Fu} \mathrm{San',}$ and determine the relationships between climatic variables, temperature, and daylength, and days to harvest for maximum marketable yield (DMMY) in individual cultivars in high tunnels. Nine cultivars were grown in high tunnels in the spring and winter of 2008 and summer of 2009 to evaluate growth and maximum marketable yield (MMY), the latter being defined as the aboveground fresh weight of $5 \pm 0.7 \mathrm{~cm}$ of plant stem. Romaine lettuce 'Jhih $\mathrm{Li}$ Wo' had a higher growth rate during the initiation of plant growth in the spring of 2008. 'Jhih Li Wo' and Batavia lettuce 'Fu San' also showed higher growth rate before harvest for the MMY (GRBHD) and exhibited higher MMY and DMMY than butterhead lettuce and leaf lettuce cultivars under summer and winter regimes. However, landraces of leaf lettuce are the main lettuces grown in high tunnels in summer rather than 'Fu San' and 'Jhih Li Wo' due to their needing fewer DMMY and having a more upright growth form. Among nine cultivars studied, Batavia lettuce 'Fu San', romaine lettuce 'Jhih Li Wo', and landrace 'Bai Yeh Wo' were found to be more adaptable to summer weather. Genotypes with superior growth and yield traits are essential for not only production but also breeding. A new cultivar, Taoyuan No.3, was developed by introducing the high growth rate trait during the initial period of plant growth from romaine lettuce 'Jhih Li Wo' into high-yielding Batavia lettuce 'Fu San'. Another experiment was performed over eight successive seasons to analyze the correlation of temperature and daylength on DMMY for each cultivar using multiple regression analysis from 2008 to 2009 . This showed that the proposed models expressed as coefficients of multiple determinants $\left(R^{2}\right)$ accounted for $72 \%$ to $\mathbf{9 1 \%}$ of the total variation in DMMY in each cultivar. Temperature affected DMMY the most and the relative contributions of temperature and daylength to DMMY differed with cultivar. These results provide information about production practices for growers in subtropical regions to use in choosing suitable lettuce cultivars.
\end{abstract}

Lettuce (Lactuca sativa L.) is a major freshly consumed vegetable and commonly used in salads worldwide (Mou, 2008). In Taiwan, lettuce is one of the most important

Received for publication 20 Oct. 2014. Accepted for publication $24 \mathrm{Feb} .2015$.

We thank T.C. Tsai and R.M. Hsu for their technical assistance on this work.

${ }^{1}$ Corresponding author. E-mail: hflo@ntu.edu.tw. leafy green vegetables and is primarily consumed cooked. The production of lettuce has increased in recent years due to it having fewer pests and diseases than other leafy vegetables grown under local hot and humid climates. This is especially true for production in high tunnels, which provide rain shelter, wind protection, and temperature moderation during severe weather conditions (Lamont, 2009; Lamont et al., 2002; Reeves and Drost, 2012). High tunnels exclude some insect pests (Lamont, 2005) and reduce foliar diseases via rain protection (Orzolek et al., 2004). Through lower disease and pest incidence and improved disease and pest management, high tunnels enhance yield and crop quality (Carey et al., 2009; Lamont, 2005; Waterer, 2003; Wells and Loy, 1993). Air and soil temperatures in high tunnels are higher than in adjacent fields, allowing crops to be planted early, mature faster, and even overwinter in some climates (Both et al., 2007; Kadir et al., 2006; Lamont, 2005; Rader and Karlsson, 2006; Reiss et al., 2004). However, the raised temperatures under high tunnels during warm seasons in subtropical regions can increase the possibility of premature bolting in lettuce. Bolting, the transition from vegetative growth to reproductive growth, begins with visually accelerated and sustained rapid elongation of the stem (Waycott, 1995). Bolting is initiated by long photoperiods (Bremer, 1931; Rappaport and Wittwer, 1956a; Wiebe and King, 1985) or high temperatures (Fukuda et al., 2009; Thompson and Knott, 1933), and is accelerated by high temperatures (Rappaport and Wittwer, 1956b). Rapid elongation of the lettuce stem represents the end of its marketability and severely affects yield and quality. Higher temperatures enhance rapid stem elongation (Fukuda et al., 2012; Rader and Karlsson, 2006; Zhao et al., 2003), hence lettuce grown in high tunnels bolts more quickly. Moreover, there is great variation among cultivars (Zhao and Carey, 2009). The response in lettuce growth to temperature depends on cultivar (Al-harbi, 2001). Some effects of environment on premature bolting by genotype were reported for crisphead lettuce (Jenni and Yan, 2009).

Premature bolting is accelerated by heat and long photoperiods. Therefore, to improve lettuce marketing and production management, models developed for predicting harvest dates based on weather variables are reported by Dufault et al. (2009), Harwood et al. (2010), Kristensen et al. (1987), and Wur et al. (1992). Dufault et al. (2009) determined the effect of diverse field temperature regimes on the quality of six commercial romaine lettuce cultivars with 22 field plantings over a 3 -year period and were able to precisely predict yield and head quality. The accurate prediction of harvest dates would minimize economic losses for growers and retailers (Harwood et al., 2010) and enable growers to schedule plantings and harvests better.

Driven by consumers' great demand for local leafy green vegetables the year around, growers in Taiwan are increasingly interested in organic and conventional lettuce production in high tunnels. Because of the local subtropical climate, with its longest photoperiod being $13 \mathrm{~h}$ and $40 \mathrm{~min}$, year-round producers should select appropriate cultivars depending on planting season to avoid yield losses from bolting. In addition, cultivars with higher growth rates allow farmers to 
increase their multiple cropping indexes in high-tunnel production, thus increasing and stabilizing profits. Therefore, this research aims to characterize the growth of commonly grown subtropical lettuce cultivars in high tunnels during all four seasons of the year to a) confirm the feasibility of developing a new high-yield, high-growth cultivar and b) determine the relationships between climatic variables, temperature, and daylength, and the number of DMMY so that growers can predict the number of days to grow for MMY. This will also assist growers in choosing appropriate cultivars according to climatic conditions.

\section{Materials and Methods}

Plant materials and cultural practices. Nine cultivars commonly grown in Taiwan representing four types of lettuce (leaf, butterhead, romaine, and crisphead subtype Batavia) were used for experiments. Seeds of leaf lettuce 'Huang Wo' and 'Jian Wo' were obtained from local farmers. Seeds of leaf lettuce cultivars Bai Yeh Wo, Chien Yeh Wo, and Yuan Yeh Wo plus romaine lettuce 'Jhih $\mathrm{Li} \mathrm{Wo}$ ' and Batavia lettuce 'Fu San' were obtained from Hsin-Sher Seed Co. Seeds of leaf lettuce 'Grand Rapids TH' were obtained from Known-You Seed Co. Seeds of butterhead lettuce 'Bibb' were obtained from Johnny's Selected Seed. Apart from 'Grand Rapids TH' and 'Bibb', all other cultivars were landraces. Among the nine cultivars studied, 'Fu San' is currently the most popular, 'Bibb' is widely grown in plant facto-

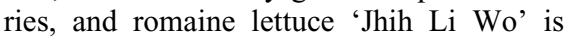
cultivated as a leaf lettuce in Taiwan.

Seeds of lettuce cultivars used for all experiments were sown into 128-cell trays containing BVB 7A medium (Bas Van Buuren, Visser, Netherlands) in a temperature-regulated high tunnel. Thereafter, seedlings with three true leaves were transplanted into $85-\mathrm{cm}$-wide quadruple-row beds with commercial spacing of $20 \mathrm{~cm}$ between plants and $20 \mathrm{~cm}$ between rows in high-tunnel experiments or into $12.5-\mathrm{cm}$ red plastic pots filled with BVB 6D growth medium (Bas Van Buuren) in growth chamber experiments. In high-tunnel experiments, lettuce plants were grown under local commercial management practices. Sprinkler and furrow irrigation were applied during early and late stages of experiments, respectively. In growth chamber experiments, each plant was irrigated by hand once daily with $100 \mathrm{~mL}$ of tap water and fertilized once biweekly with 200 $\mathrm{mg} \cdot \mathrm{L}^{-1}$ of a $20 \mathrm{~N}-8.7 \mathrm{P}-16.6 \mathrm{~K}$ water-soluble fertilizer (Peters 20-20-20; Scotts Co., Marysville, $\mathrm{OH})$.

Experiments were conducted in high tunnels or growth chambers at the Taoyuan District Agricultural Research and Extension Station (TYDARES, Taiwan) from 2008 to 2013. The experimental site is located at $121^{\circ} 01^{\prime} 37.9^{\prime \prime} \mathrm{E}$ longitude, $24^{\circ} 57^{\prime} 17.7^{\prime \prime} \mathrm{N}$ latitude, and $33.5 \mathrm{~m}$ above mean sea level.
Temperature and light intensity were recorded with a HOBO Pendant ${ }^{\circledR}$ temperature/ light data logger (HOBO H8-032-08; Onset Computer Corporation, MA) and LI-250 light meter (LI-COR, Lincoln, NE).

Plant growth in 2008 high-tunnel assessment. Seeds of the nine cultivars described above were sown on 21 Mar. Seedlings were transplanted on $10 \mathrm{Apr}$. in high tunnels and local commercial growing methods were followed for $44 \mathrm{~d}$ after transplanting (DAT). A randomized complete block design with three replicates was applied. Aboveground fresh weight and stem length were measured from five plants selected per plot at 3- to 7-d intervals from 22 DAT. The air in the high tunnels had an average daily maximum temperature, minimum temperature, and mean temperature of $30.5,17.3$, and $25.2{ }^{\circ} \mathrm{C}$, respectively, with $5 \mathrm{~d}$ of temperature exceeding $30^{\circ} \mathrm{C}$. The average daily maximum light intensity, minimum light intensity, and mean light intensity were 864.6, 115.1 , and $399.6 \mu \mathrm{mol} \cdot \mathrm{m}^{-2} \cdot \mathrm{s}^{-1}$, respectively. Daylength ranged from $12 \mathrm{~h} 30 \mathrm{~min}$ to $13 \mathrm{~h}$ $30 \mathrm{~min}$.

High-tunnel yield during Winter 2008 and Summer 2009. Seeds of the nine cultivars described above were sown on 26 Dec. 2008 in winter and 26 June 2009 in summer. Seedlings were transplanted on $18 \mathrm{Jan}$. 2009 in winter and 15 July 2009 in summer using local high-tunnel commercial growing methods. A randomized complete block design was applied with four replicates. Harvest was performed on the day that stem length reached $5 \pm 0.7 \mathrm{~cm}$, the maximum locally marketable stem length for lettuce. The MMY was considered to be the aboveground fresh weight when stem length reached $5 \pm 0.7 \mathrm{~cm}$. Aboveground fresh weight and stem length of 15 plants selected per plot at harvest were measured. The DAT to harvest for each cultivar was calculated as DMMY. During the experimental period, the average daily maximum temperature, minimum temperature, and mean temperature were 24.5 , 10.4 , and $18.4{ }^{\circ} \mathrm{C}$ in winter and $32.9,26.6$, and $31.4{ }^{\circ} \mathrm{C}$ in summer, respectively. The average daily maximum light intensity, minimum light intensity, and mean light intensity were $343.7,41.8$, and $153.4 \mu \mathrm{mol} \cdot \mathrm{m}^{-2} \cdot \mathrm{s}^{-1}$ in winter and $774.7,153.3$, and $475.6 \mu \mathrm{mol} \cdot \mathrm{m}^{-2} \cdot \mathrm{s}^{-1}$ in summer, respectively. Daylength ranged from $10 \mathrm{~h} 30 \mathrm{~min}$ to $12 \mathrm{~h}$ in winter and $13 \mathrm{~h}$ to $13 \mathrm{~h} 40 \mathrm{~min}$ in summer.

Feasibility of integrating plant growth characteristics for developing a new hightunnel cultivar. 'Taoyuan No.3' originated from a cross between romaine lettuce 'Jhih $\mathrm{Li}$ Wo' and Batavia lettuce 'Fu San' during Summer 2006 using the clip-and-wash method of emasculation (Nagata, 1992). Following the cross, modified single-seed descent (Fehr, 1987) method was applied from $F_{2}$ to $F_{6}$ generation and elite lines were selected at $\mathrm{F}_{6}$ for further evaluation. 'Taoyuan No.3' was developed based on the performance in the field trials. "Taoyuan No.3' and its parents were used to evaluate plant growth under high-tunnel conditions. Seeds of 'Taoyuan No.3', 'Jhih Li Wo', and 'Fu San' were sown on 22 Feb. 2013 and seedlings were transplanted on 14 Mar. 2013 in high tunnels following local commercial growing methods. A randomized complete block design was applied with four replicates. Five plants selected per plot at 7-d intervals from 14 DAT were evaluated for aboveground fresh weight and stem length. During the experimental period, the average daily maximum temperature, minimum temperature, and mean temperature were 25.1, 14.6, and $19.8^{\circ} \mathrm{C}$, respectively. Daylength ranged from 12 to $13 \mathrm{~h}$.

Contributions of temperature and daylength to yield under high-tunnel conditions. Experiments were conducted in high tunnels during eight successive seasons from 2008 to 2010 . Seeding dates, transplanting dates, and mean daily temperatures were shown in Table 1 and followed local commercial growing methods. Various planting seasons represented the various temperature regimes. The mean daily temperature from planting to harvest was measured and considered as the temperature treatment in this experiment.

Photoperiod treatments consisted of a short-day period (SD, $10 \mathrm{~h}$ ), a mediumlength day period (MD, $13 \mathrm{~h}$ ), and a long-day period (LD, $15 \mathrm{~h})$. The short photoperiod was provided by covering plants with black cloth at 5:30 PM and removing the black cloth at 7:30 AM the next morning. Medium and long photoperiods were achieved by providing supplemental light from 5:30 AM to sunrise and from sunset to 6:30 PM for MD treatment or 8:30 PM for LD treatment. Supplemental light controlled by timers was applied by $500-\mathrm{W}$ fluorescent bulbs with light intensity of $11.3 \mu \mathrm{mol} \cdot \mathrm{m}^{-2} \cdot \mathrm{s}^{-1}$. The experimental design was a split-split plot (Montgomery, 1984) with four replicates. Planting seasons representing growing temperature regimes were assigned to main plots, daylength regimes to subplots, and cultivars to subsubplots. Harvest dates were recorded when plant stem lengths reached $5 \pm 0.7 \mathrm{~cm}$ and DATs to harvest for each cultivar calculated to evaluate the DMMY.

Effects of temperature and daylength on stem growth in growth chambers. Four cultivars (leaf lettuces Jian Wo and Chien Yeh

Table 1. Seeding dates (SDD) and transplanting dates (TPD) of lettuce and mean daily temperature (MDT) in eight successive seasons from 2008 to 2010 .

\begin{tabular}{|c|c|c|c|c|c|c|}
\hline \multirow[b]{2}{*}{ Planting season } & \multicolumn{3}{|c|}{ First yr } & \multicolumn{3}{|c|}{ Second yr } \\
\hline & SDD & TPD & $\operatorname{MDT}\left({ }^{\circ} \mathrm{C}\right)$ & SDD & TPD & $\operatorname{MDT}\left({ }^{\circ} \mathrm{C}\right)$ \\
\hline Autumn & 1 Sept. 2008 & 20 Sept. 2008 & $26.0-27.1$ & 28 Aug. 2009 & 17 Sept. 2009 & $26.3-28.1$ \\
\hline Winter & 26 Nov. 2008 & 15 Dec. 2008 & $16.4-17.4$ & 1 Dec. 2009 & 20 Dec. 2009 & $17.5-17.9$ \\
\hline Spring & 12 Mar. 2009 & 3 Apr. 2009 & $23.1-24.0$ & 18 Mar. 2010 & 6 Apr. 2010 & $22.4-24.3$ \\
\hline Summer & 22 June 2009 & 10 July 2009 & $29.5-30.1$ & 25 June 2010 & 13 July 2010 & $31.4-31.6$ \\
\hline
\end{tabular}


Wo, romaine lettuce Jhih Li Wo, and Batavia lettuce Fu San) were used for the experiments. Four identical growth chambers (modified Fame F-560DN; Luen Fat Enterprise) were set to different day/night $(\mathrm{D} / \mathrm{N})$ temperatures $\left(31 / 28{ }^{\circ} \mathrm{C}, 28 / 25^{\circ} \mathrm{C}, 25 / 23{ }^{\circ} \mathrm{C}\right.$, and $20 / 15^{\circ} \mathrm{C}$ ) with 15 (LD, long-day treatment), 13 (MD, medium-long day treatment), and $10 \mathrm{~h}$ (SD, short-day treatment) photoperiods, respectively. Light intensity at 310 $\mu \mathrm{mol} \cdot \mathrm{m}^{-2} \cdot \mathrm{s}^{-1}$ was provided by light-emitting diodes. The various temperature and photoperiod regimes in growth chambers simulated high-tunnel conditions during Taiwan's four seasons. Seeds were sown on 16 Jan. 2011 for SD regimes, 31 May 2011 for LD regimes, and 8 Aug. 2011 for MD regimes. Seedlings were transplanted on 5 Feb. 2011 for SD regimes, 17 June 2011 for LD regimes, and 7 Sept. 2011 for MD regimes. A completely randomized design was applied with three replicates. Four plants per plot were used to measure stem length at 2-d intervals after transplanting and on the dates when plant stem length reached $5 \mathrm{~cm}$ to calculate each cultivar's DMMY.

Statistical analysis. All data collected from high-tunnel and growth chamber experiments were analyzed using the PROC GLM procedure of SAS (SAS Institute, Cary, NC), and means were separated by Fisher's least significant difference test at the 5\% level of probability. Pearson's correlation analysis was used to identify the relationship between MMY and DMMY. Multiple linear regression analysis was used to analyze the differences among cultivars in the sensitivity of the DMMY of lettuce plants to temperature and daylength. The relative contribution of temperature and daylength to DMMY was determined using standardized partial regression coefficients and the overall strength of the relationships was quantified with multiple correlation coefficients. All graphs were constructed by Sigmaplot 9 (Systat Software, San Jose, CA).

\section{Results and Discussion}

Plant growth and yield in high-tunnel experiments. Among the nine cultivars studied, romaine lettuce 'Jhih Li Wo' exhibited higher aboveground fresh weight than other cultivars until 33 DAT, and Batavia lettuce 'Fu San' exhibited the lowest aboveground fresh weight on all sampling dates. Stem length in all cultivars studied reached or exceeded the stage of MMY at 33 DAT except for 'Fu San', which reached it on 44 DAT. Meanwhile, the MMY in 'Fu San' was higher than in the other cultivars (Fig. 1). Thus, 'Fu San' exhibited the highest DMMY and MMY among all cultivars. Moreover, 'Jhih Li Wo' had a higher growth rate during plant growth initiation and 'Fu San' grew more slowly than other cultivars during their entire spring growth.

Yields during Winter 2008 and Summer 2009 were significantly different among planting seasons, cultivars, and their interactions for MMY, DMMY, and GRBHD as evidenced by highly significant effects on the model (Table 2). All cultivars studied showed the same increasing trend over the two seasons. The MMY and DMMY in all cultivars studied under winter conditions were significantly higher than in plants grown in summer, ranging $192.3 \%$ to $442.4 \%$ and $196.8 \%$ to $274.7 \%$, respectively. The GRBHD of all cultivars grown in winter was also significantly higher than in plants under summer conditions except for 'Huang Wo' and 'Bai Yeh Wo', which were not significantly different between summer and winter (Table 3). The effects of cultivar choice on MMY, DMMY, and GRBHD were significantly affected by planting season. The MMY, DMMY, and GRBHD of nine lettuce cultivars ranged 26.9-57.7 g/plant in summer and $74.8-198.3 \mathrm{~g} /$ plant in winter, 18.2-27.1 DAT in summer and 43.5-62.0 DAT in winter, and $1.37-2.50 \mathrm{~g} \cdot \mathrm{d}^{-1}$ in summer and $1.72-3.20 \mathrm{~g} \cdot \mathrm{d}^{-1}$ in winter. The MMY in ' $\mathrm{Fu}$ San' was significantly higher than in all other cultivars over two seasons, and the lowest MMY was obtained in butterhead lettuce 'Bibb'. There were no significant differences among Bibb, leaf lettuce Jian Wo, Chien Yeh Wo, and Grand Rapids TH cultivars during summer. However, due to the obviously higher relative increase in MMY during winter in 'Bibb' and 'Jian Wo' than in other cultivars, the MMYs for 'Bibb' and 'Jian Wo' were not the lowest in winter. On the contrary, the MMY of 'Jian Wo' and 'Jhih Li Wo' was ranked second highest among all cultivars studied under winter conditions, following Batavia lettuce 'Fu San'. Likewise, the lowest MMY in winter was obtained in leaf lettuce 'Huang Wo' due to it having the lowest relative MMY increase in winter among all cultivars studied, although 'Huang Wo' MMY was not the lowest in summer. 'Huang Wo' DMMY showed similar trends.

Among all cultivars studied, the DMMY in 'Fu San' was significantly higher than in other cultivars over two seasons. 'Bibb' exhibited the lowest DMMY in summer, and the difference was significant except in comparison with leaf lettuce 'Bai Yeh Wo', 'Chien Yeh Wo', and 'Yuan Yeh Wo'. DMMY in 'Chien Yeh Wo' was also significantly lower in winter than all other cultivars except for 'Huang Wo'. 'Bai Yeh Wo' had the highest GRBHD in summer, which was significantly different from other cultivars except 'Jhih Li Wo' and 'Fu San' (Table 3). In winter, GRBHD also differed significantly among cultivars, the highest being in ' $\mathrm{Fu}$ San' and the lowest in 'Huang Wo'. The highest GRBHD in 'Bai Yeh Wo' resulted in its higher MMY than other leaf lettuce cultivars in summer, although its DMMY was ranked among the lowest. Thus, besides DMMY, the GRBHD of lettuce plants was another factor dominating yield. Consequently, the highest DMMY and GRBHD

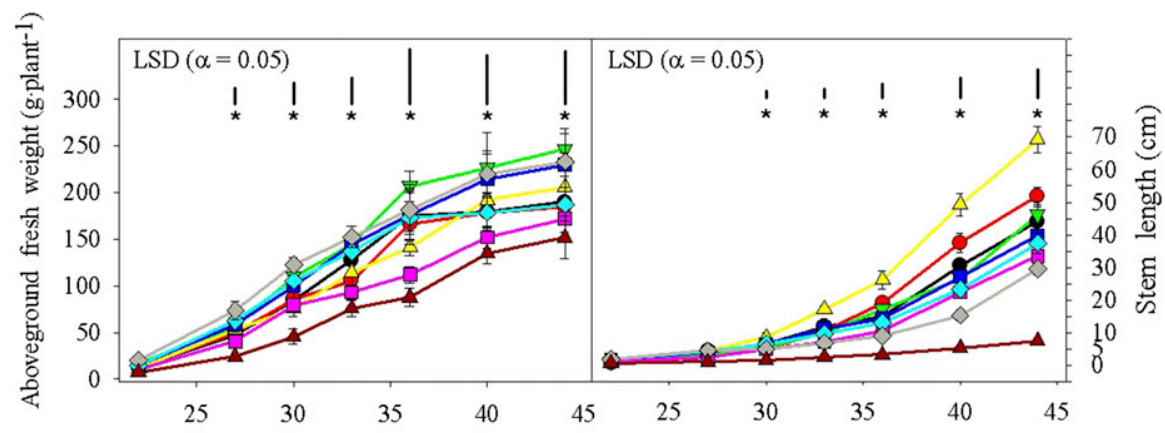

Days after transplanting
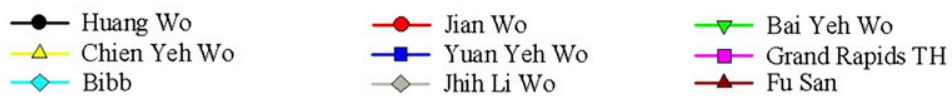

Fig. 1. Daily aboveground fresh weight and stem length of nine lettuce cultivars in spring. Sown on $22 \mathrm{Mar}$ 2008. Transplanted on 11 Apr. 2008. Data are means \pm SE of three replicates of five plants each. Asterisks denote significant differences among cultivars at $P \leq 0.05$ according to one-way analysis of variance and bars represent least significant difference value according to Fisher's least significant difference test at $P \leq 0.05$, within sampling dates.

Table 2. Analysis of variance for the effects of cultivar and planting season on the maximum marketable yield (MMY), days to harvest for maximum marketable yield (DMMY), and growth rate before harvest day for maximum marketable yield (GRBHD) in lettuce plants grown in high tunnels.

\begin{tabular}{lrrrr}
\hline & & \multicolumn{3}{c}{ Mean square } \\
\cline { 3 - 5 } Source of variation & df & MMY & DMMY & GRBHD \\
\hline Block & 3 & $38.9^{\text {NS }}$ & $3.6^{\text {NS }}$ & $0.04^{\text {NS }}$ \\
Planting season (PS) & 1 & $111,292.4^{* * *}$ & $12,194.0^{* * *}$ & $6.11^{* * *}$ \\
Cultivar (C) & 8 & $883.6^{* * *}$ & $150.3^{* * *}$ & $0.97^{* * *}$ \\
PS $\times$ C & 8 & $1,618.3^{* * *}$ & $36.4^{* * *}$ & $0.39^{* * *}$ \\
Error & 51 & 64.5 & 3.8 & 0.07 \\
\hline
\end{tabular}

Ns, ***Nonsignificant or significant at $P \leq 0.001$, respectively. 


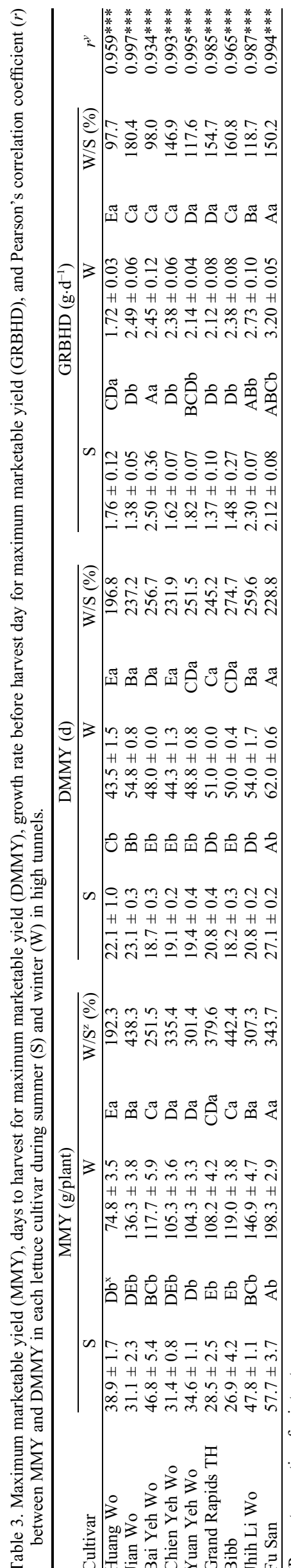

resulted in the highest MMY being in ' $\mathrm{Fu}$ San' among all cultivars studied over two seasons and the lowest DMMY and GRBHD resulted in the lowest MMY in 'Huang Wo' among all cultivars studied under winter conditions.

The optimum growing temperature for lettuce is $18.5{ }^{\circ} \mathrm{C}$, and flower initiation generally occurs between 21 and $27{ }^{\circ} \mathrm{C}$ (Wallace et al., 2012). The transition to flowering in lettuce begins with rapid elongation of the stem as a result of increasing daylength and temperature (Dufault et al., 2006; Waycott, 1995). In this experiment, winter was characterized by cooler temperatures and shorter daylengths, suitable for lettuce growth. In contrast, summer heat and long days resulted in low MMY in all cultivars studied. Although the lowest yields per plant were produced in summer, lettuce retained its local marketability because of the shortage of green leafy vegetables in summer (unpublished data). Due to fewer DMMY and greater uprightness in all leaf lettuce landraces studied, higher plant density was usually adopted to increase local lettuce yields in summer. Therefore, leaf lettuce landraces were the main types of lettuce cultivated in high tunnels in summer, excluding Batavia lettuce 'Fu San' and romaine lettuce 'Jhih $\mathrm{Li}$ Wo'. In addition, among the nine cultivars studied, Batavia lettuce 'Fu San', romaine lettuce 'Jhih Li Wo', and leaf lettuce 'Bai Yeh Wo' were more adaptable to summer conditions.

Batavia lettuce 'Fu San' and romaine lettuce 'Jhih Li Wo' exhibited higher MMY and DMMY than butterhead and leaf lettuce (Fig. 1; Table 3). Romaine and leaf lettuce showed higher plant growth rate during the initiation period of plant growth than butterhead and Batavia lettuce; however, Batavia lettuce 'Fu San', as well as romaine lettuce 'Jhih Li Wo', exhibited the highest GRBHD. Therefore, the fact that individual cultivars possess differing expressions of beneficial yield traits suggests that the improvement of MMY and GRBHD in lettuce cultivars is feasible and would be efficient via hybridization between different types of lettuce.

Lettuce is a quantitative long-day plant (Bremer, 1931), with rapid stem elongation following the transition from vegetative to reproductive growth being induced by high temperatures (Fukuda et al., 2009; Thompson and Knott, 1933) and long photoperiods (Bremer, 1931; Rappaport and Wittwer, 1956a; Waycott, 1995; Wiebe and King, 1985). In this experiment, the responses of MMY in cultivars to planting seasons, which combine the effects of temperature and photoperiod, varied significantly. Therefore, subsequent experiments were executed to examine the contributions of these factors

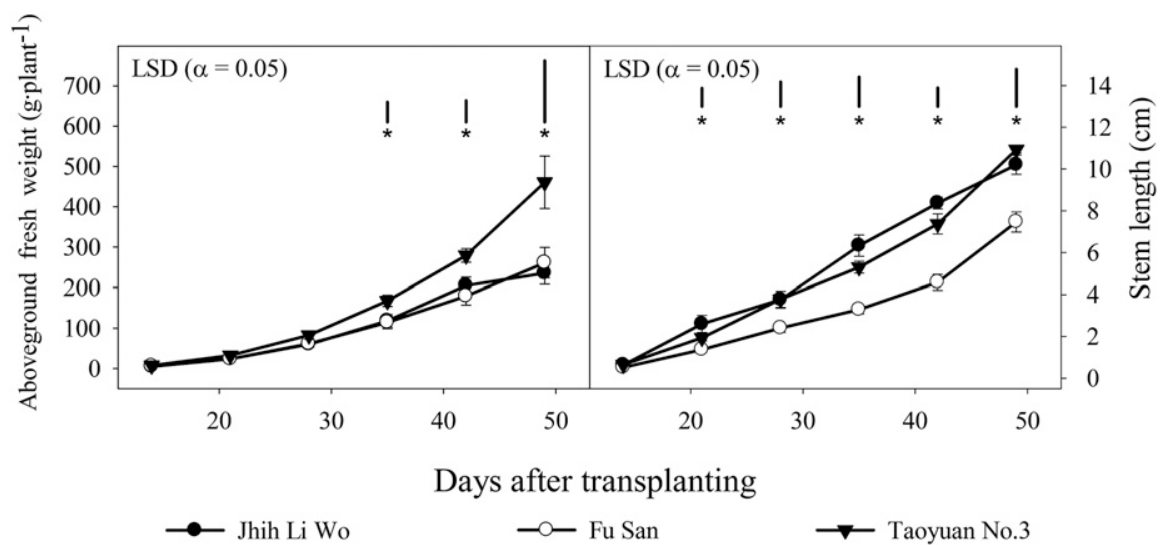

Fig. 2. Daily aboveground fresh weight and stem length of lettuce 'Taoyuan No.3' and its parental materials. Sown on $22 \mathrm{Feb} .2013$, and transplanted on 14 Mar. 2013. Data are means $\pm \mathrm{sE}$ of four replicates of five plants each. Asterisks denote significant differences among cultivars at $P \leq 0.05$ according to one-way analysis of variance and bars represent least significant difference value according to Fisher's least significant difference test at $P \leq 0.05$, within sampling dates.

Table 4. Analysis of variance for the effects of temperature, daylength, and cultivar on the number of days to harvest for maximum marketable yield (DMMY) in lettuce plants grown in high tunnels.

\begin{tabular}{lrrrrr}
\hline Source of variation & \multicolumn{1}{c}{ df } & Sum of squares & Mean square & F value & $P>\mathrm{F}$ \\
\hline Block (B) & 3 & 0.1 & 0.02 & 0.4 & 0.7859 \\
Temperature (T) & 7 & $103,676.4$ & $14,810.91$ & $335,006.0$ & $<0.0001$ \\
Whole plot error & 21 & 0.9 & 0.04 & & \\
Daylength (DL) & 2 & $10,095.6$ & $5,047.79$ & $9,641.1$ & $<0.0001$ \\
T $\times$ DL & 14 & $3,759.5$ & 268.54 & 512.9 & $<0.0001$ \\
Subplot error & 48 & 25.1 & 0.52 & & \\
Cultivar (C) & 8 & $35,685.2$ & $4,460.65$ & $1,260.0$ & $<0.0001$ \\
$\mathrm{~T} \times \mathrm{C}$ & 56 & $13,086.4$ & 233.69 & 66.0 & $<0.0001$ \\
DL $\times$ C & 16 & $1,870.2$ & 116.89 & 33.0 & $<0.0001$ \\
T $\times$ DL $\times$ C & 112 & $1,898.7$ & 16.95 & 4.8 & $<0.0001$ \\
Sub-subplot error & 576 & $2,039.2$ & 3.54 & & \\
Corrected total & 863 & $172,137.2$ & & & \\
\hline
\end{tabular}


to the MMY in each cultivar and separate the effects of temperature and photoperiod. In addition, Pearson's correlation coefficients $(r)$ show a highly significant positive correlation between DMMY and MMY in all cultivars (Table 3). Therefore, the evaluation of DMMY in lettuce plants could generally be used to infer MMY responses in plants of the same cultivar.

Feasibility of integrating plant growth characteristics for developing a new cultivar for high-tunnel use. The daily aboveground fresh weight of 'Taoyuan No.3' was consistently higher than that of its parents during the entire period of vegetative growth, and the GRBHD in 'Taoyuan No.3' was higher than in 'Fu San' (Fig. 2). A breeding program must start with an assessment of existing genetic variation in the germplasm to find the sources of beneficial traits (Lafta and Mou, 2013; Mou, 2005). Genotypes assessed as having desired traits could be used as parents to develop superior cultivars. The above-described experiment indicated that Batavia lettuce 'Fu San' possesses the highest MMY but the lowest growth rate during the initial period of plant growth. Thus, our studies provide evidence that introducing the high growth rate trait during the initial period of plant growth from romaine lettuce 'Jhih $\mathrm{Li}$ Wo' to the high yielding Fu San cultivar is feasible for developing a new cultivar with early maturity and high yields.

Contributions of temperature and daylength to yield and growth. Our analysis revealed that lettuce plant DMMY was significantly influenced by temperature, daylength, cultivar, and their interactions (Table 4). The sum of squares (SS) in treatments was proportioned to $60.2 \%$ of temperature SS, $5.9 \%$ of daylength SS, and $20.7 \%$ of cultivar SS. A large variation explained by temperature suggests that the effects of temperature on lettuce plant DMMY is stronger than other factors. The impact of temperature on lettuce plant DMMY is also reflected in the significant interactions between cultivars and daylength.

The effect of daylength on lettuce plant DMMY for nine individual cultivars in eight successive planting seasons representing various temperature treatments is shown in Fig. 3. Lettuce plant DMMY under most temperature regimes was the longest in SD treatments for all cultivars, and the difference was significant except for Batavia lettuce ' $\mathrm{Fu}$ San'. The differences in DMMY among daylength treatments for nine individual cultivars showed a decreasing trend from low temperatures to high temperatures, indicating a stronger effect of daylength on DMMY under low-temperature regimes.

The DMMY of each cultivar during each season in the first year was longer than in the second year due to lower temperatures during the first year (Fig. 3). Daily temperature data collected at the experimental site with data loggers during the experiments (Fig. 4) shows that temperature patterns varied greatly and with large fluctuations among the four seasons. For the same season between two successive years, temperatures were lower in the first year than in the second.

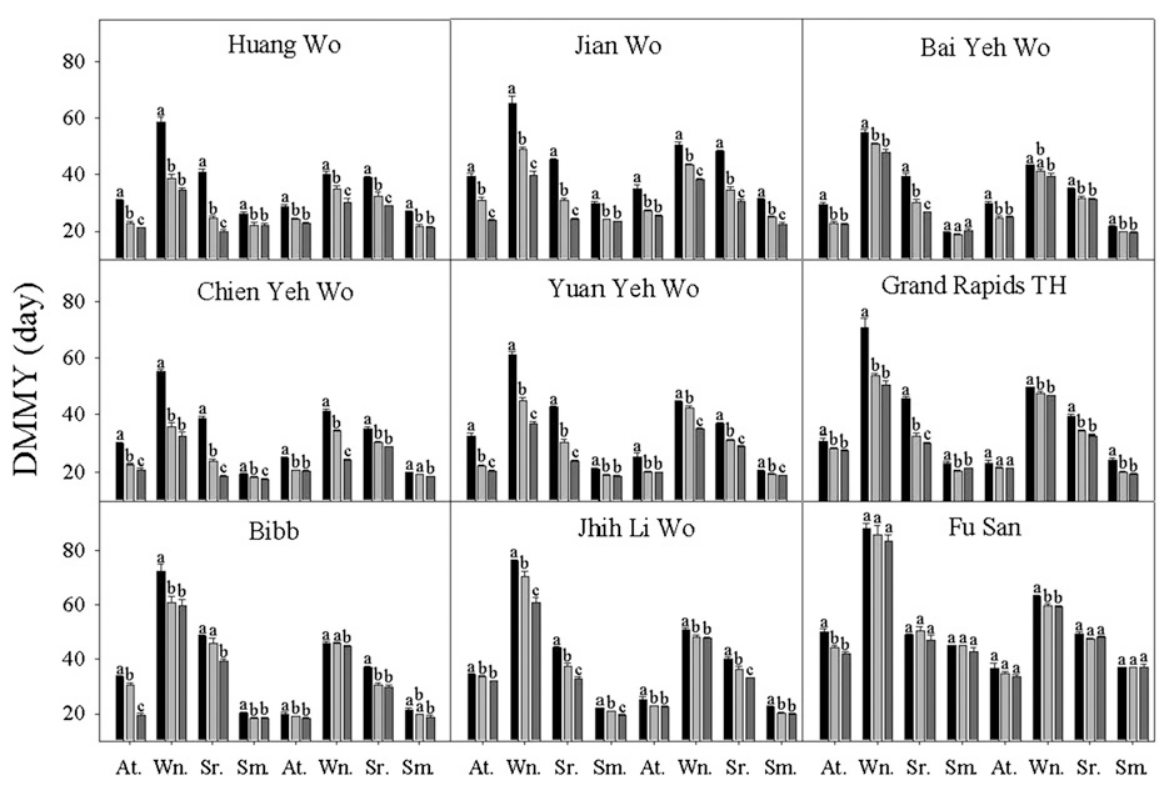

Planting season

$10 \mathrm{~h}$

ए $13 \mathrm{~h}$

$15 \mathrm{~h}$

Fig. 3. Effects of daylength on the number of days to harvest for the maximum marketable yield (DMMY) in nine lettuce cultivars in eight successive planting seasons. At. for autumn, Wn. for winter, Sr. for spring, and $\mathrm{Sm}$. for summer. Data are means $\pm \mathrm{SE}(\mathrm{n}=4)$. Means within season topped by the same letter are not significantly different at $P \leq 0.05$ according to Fisher's least significant difference test.

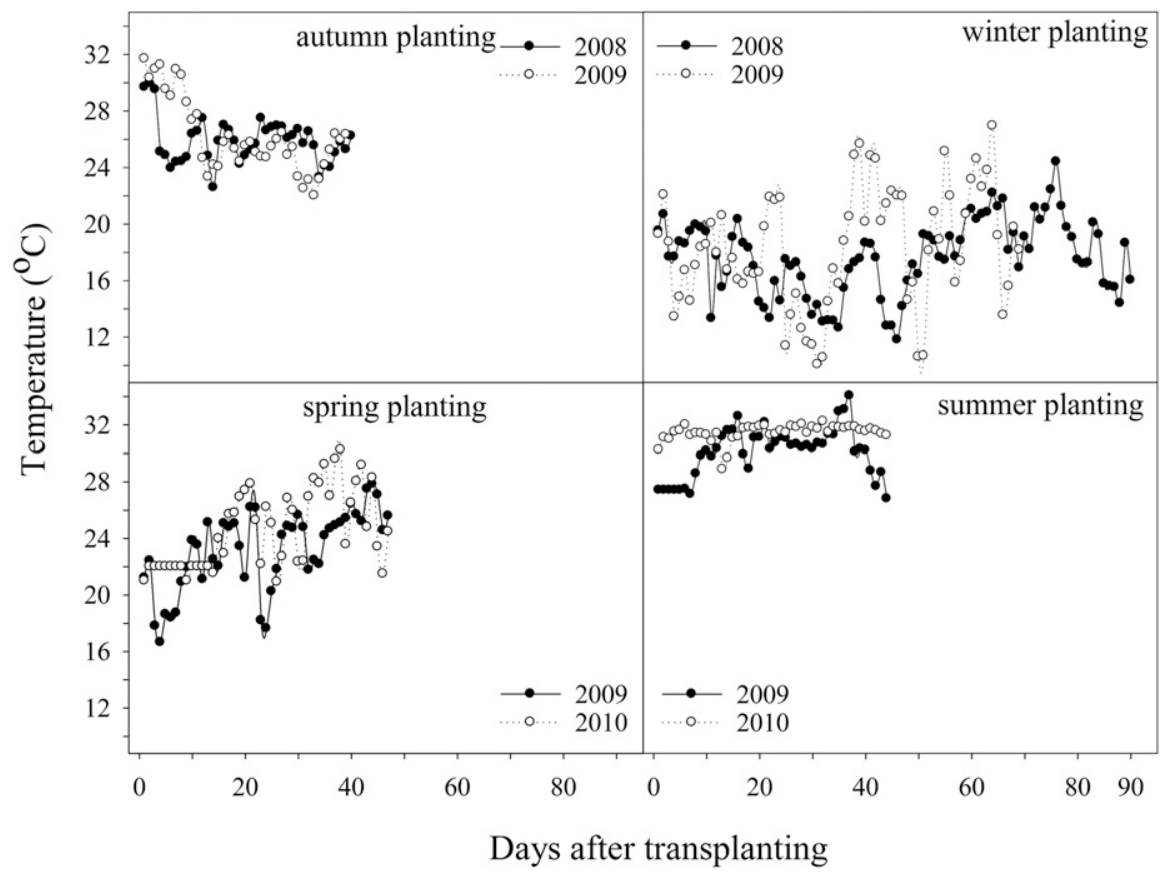

Fig. 4. Daily mean temperature in high tunnels during the experiment.

Batavia lettuce 'Fu San' consistently exhibited the highest DMMY among all cultivars studied under same daylength and temperature conditions. The ranking order of other cultivars was influenced intricately by the interaction of temperature and daylength. Therefore, multiple regression analysis (Neter et al., 1989) was used to analyze the relationships between these factors and DMMY.
Multiple regression equations were solved to explain the correlations of temperature and daylength to DMMY on the basis of means collected for all nine lettuce cultivars (Table 5). The proposed models were highly significant and successfully accounted for $72 \%$ to $91 \%$ of the total variation in DMMY expressed as coefficients of multiple determinant $\left(R^{2}\right)$ for each cultivar. The remaining $9 \%$ to $28 \%$ could be due to measurement 
Table 5. Multiple regression analysis using general linear models (GLMs) for the correlation between two weather factors (DL, daylength; T, temperature) and days to harvest for maximum marketable yield (DMMY) in nine lettuce cultivars grown in high tunnels.

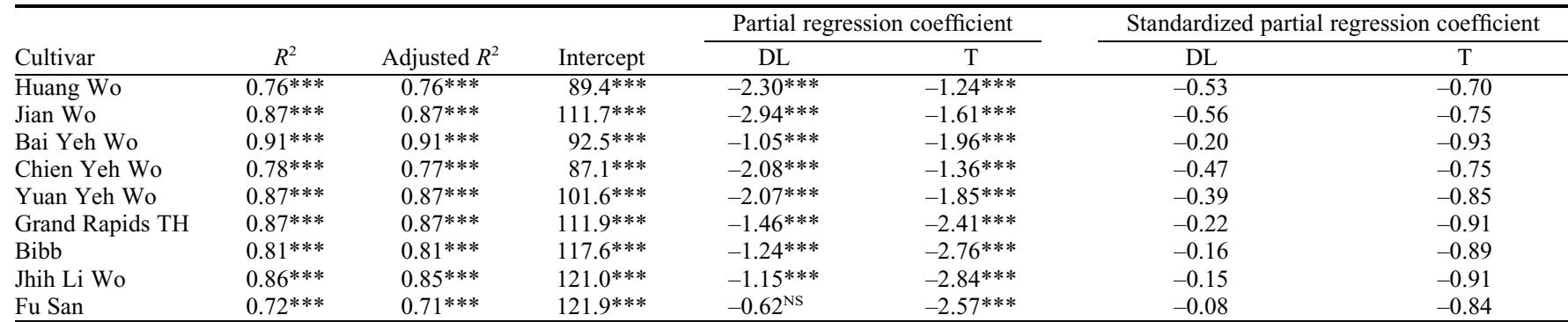

Ns, ***Nonsignificant or significant at $P \leq 0.001$, respectively. The total number of observations is 96 for each cultivar.

errors, random errors, and/or other factors besides temperature and daylength. Temperature and daylength significantly and negatively contributed to DMMY in all cultivars studied except for the effect of daylength on DMMY in 'Fu San', indicating that a higher temperature or longer day resulted in shorter DMMY. Reduced DMMY and lower yields are caused by rapid stem elongation when lettuce is grown under flower-inducible conditions (Jenni and Yan, 2009; Lee et al., 2003; Nothmann, 1977). For Batavia lettuce 'Fu San', the contribution of daylength to DMMY in lettuce plants was not significant, indicating that daylength only slightly influenced ' $\mathrm{Fu}$ San' DMMY. However, this is inconsistent with studies reported by Waycott (1995) in which photoperiod but not temperature is characterized as the critical mediator of lettuce growth rates. Waycott (1995) also reported a range of genetic responses to various daylengths among lettuce genotypes, which might explain the present results. In addition, the relative contributions of temperature and daylength to DMMY in lettuce plants differed among cultivars. As revealed by the standardized partial regression coefficients (Table 5), the contribution of temperature to DMMY in lettuce plants was higher than that of daylength in all cultivars, indicating that the response of DMMY was more sensitive to temperature swings. Thus, breeding high-temperature-tolerant cultivars with late bolting is a high priority for mitigating the effects of global warming and climate changes.

The relative contributions of temperature and daylength toward DMMY in leaf lettuce 'Huang Wo' were similar to those in leaf lettuces 'Jian Wo', 'Chien Yeh Wo', and 'Yuan Yeh Wo', and the difference between the contributions of temperature and daylength was small. The relative contributions of temperature and daylength to DMMY in leaf lettuce 'Bai Yeh Wo' were similar to that in leaf lettuce 'Grand Rapids TH', butterhead lettuce 'Bibb', romaine lettuce 'Jhih Li Wo', and Batavia lettuce 'Fu San', and the difference between the contribution of temperature and daylength was large. The contribution of daylength to DMMY in most Taiwan landraces was greater than in non-Taiwan leaf cultivars, indicating that leaf lettuce landrace yields were more sensitive to daylength except for 'Bai Yeh Wo'.

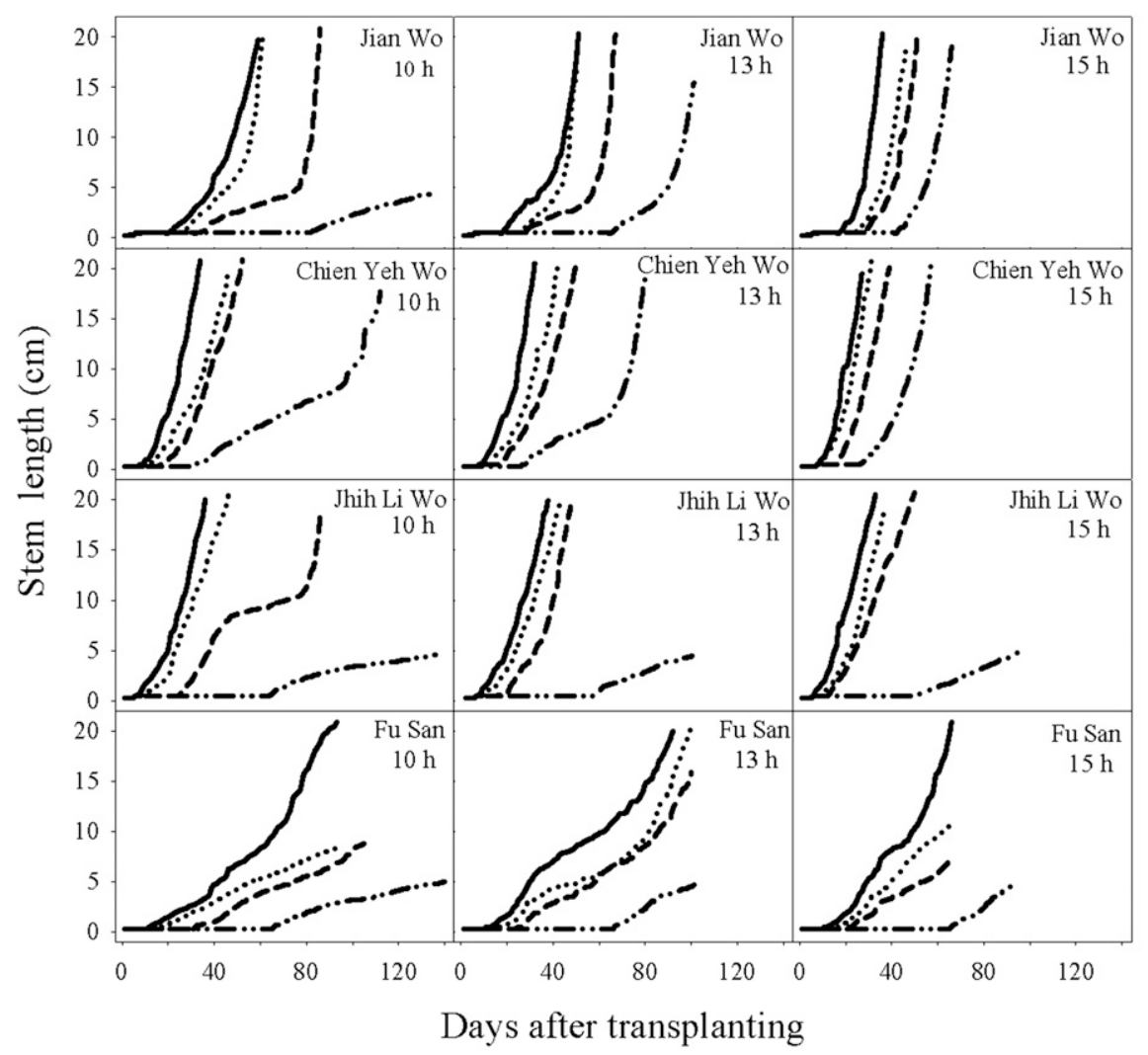

$-31^{\circ} \mathrm{C} / 28^{\circ} \mathrm{C} \quad \cdots \cdots \cdot \cdot 28^{\circ} \mathrm{C} / 25^{\circ} \mathrm{C} \quad-\cdots 25^{\circ} \mathrm{C} / 23^{\circ} \mathrm{C} \quad \cdots \cdots \cdot 20^{\circ} \mathrm{C} / 15^{\circ} \mathrm{C}$

Fig. 5. Effects of temperature on daily stem length in four lettuce cultivars cultivated in growth chambers under three different daylengths. Data are the means of five plants.

Table 6. Analysis of variance of the effects of temperature, daylength, and cultivar on the number of days to harvest for maximum marketable yield (DMMY) in lettuce grown in growth chambers.

\begin{tabular}{lrcrrr}
\hline Source of variation & df & Sum of squares & Mean square & F value & $P>$ F \\
\hline Daylength (DL) & 2 & $10,787.5$ & $5,393.8$ & 547.4 & $<0.0001$ \\
Cultivar (C) & 3 & $21,373.7$ & $7,124.6$ & 723.0 & $<0.0001$ \\
Temperature (T) & 3 & $106,016.4$ & $35,338.8$ & $3,586.2$ & $<0.0001$ \\
DL $\times$ C & 6 & $2,379.7$ & 396.6 & 40.3 & $<0.0001$ \\
$\mathrm{C} \times \mathrm{T}$ & 9 & $12,810.7$ & $1,423.4$ & 144.5 & $<0.0001$ \\
$\mathrm{DL} \times \mathrm{T}$ & 6 & $8,130.2$ & $1,355.0$ & 137.5 & $<0.0001$ \\
$\mathrm{DL} \times \mathrm{C} \times \mathrm{T}$ & 18 & $2,117.1$ & 117.6 & 11.9 & $<0.0001$ \\
Error & 96 & 946.0 & 9.9 & & \\
Corrected total & 143 & $164,561.3$ & & &
\end{tabular}

Four cultivars were raised in growth chambers to verify the effects of temperature and daylength on lettuce stem growth. Stem growth was faster at higher temperatures, and long days decreased the differences in stem growth rates among temperature regimens for all cultivars. On the other hand, the effects of daylength on stem elongation under lowtemperature regimes were stronger than under high-temperature regimes (Fig. 5). In 
addition, among the four cultivars studied, 'Fu San' alone or with some of the other cultivars had the highest DMMY under all regimes, consistent with the results of the high-tunnel experiments. Similarly, the DMMY was significantly influenced by interactions among temperature, daylength, and cultivar (Table 6). The largest proportion of variation was explained by temperature, followed by cultivar and daylength. Altogether, the growth chamber experiment confirmed the results of the high-tunnel experiments.

The interactions between genotype and environment influence lettuce plant growth (Dufault et al., 2006; Jenni and Yan, 2009). Among the environmental factors, regional climate likely plays a critical role in determining the marketable yields of lettuce (Wallace et al., 2012). Furthermore, temperature is the most important environmental factor for lettuce growth (Bierhuizen et al., 1973; Rader and Karlsson, 2006). These previous studies were supported by the results of our experiments. The correlation between the number of days required for the nine studied lettuce cultivars to grow to their MMY vs. temperature and daylength was determined. Lettuce growers might be better able to predict DMMY by using our multiple regression equations, depending on climatic conditions.

In conclusion, our studies demonstrate the growth characteristics of nine local commonly grown lettuce cultivars and suggest that Batavia lettuce 'Fu San', romaine lettuce 'Jhih Li Wo', and leaf lettuce 'Bai Yeh Wo' are the most suitable cultivars for summer production. Meanwhile, using landraces with desired traits as parental materials, a new high-yielding cultivar, Taoyuan No.3, was developed via intertype crossing. In addition, the relationships between weather factor, temperature, and daylength, and DMMY in lettuce plants were determined by multiple regression analysis. The equations might help growers choose lettuce cultivars according to climatic conditions and predict the number of days for lettuce cultivars to reach their MMY when grown in high tunnels within subtropical regions. Multiple regression can serve as a reference for setting growing temperatures and daylengths for the more efficient cultivation of lettuce in plant factories. Moreover, since the DMMY in all cultivars studied was more sensitive to temperature swings, there is a high priority for breeding hightemperature-tolerant cultivars with late bolting characteristics to mitigate the effects of global warming and climate changes. Our results provide farmers with useful information on production practices and could be beneficial for lettuce production not only in Taiwan but also in other subtropical regions.

\section{Literature Cited}

Al-harbi, A.R. 2001. Growth and flowering of five lettuce cultivars as affected by planting date. $\mathrm{J}$. Veg. Crop Prod. 7(1):23-36.
Bierhuizen, J.F., J.L. Ebbens, and N.C.A. Koomen. 1973. Effects of temperature and radiation on lettuce growing. Neth. J. Agr. Sci. 21:110-116.

Both, A.J., E. Reiss, J.F. Sudal, K.E. Holmstrom, C.A. Wyenandt, W.L. Kline, and S.A. Garrison. 2007. Evaluation of a manual energy curtain for tomato production in high tunnels. HortTechnology 17:467-472.

Bremer, A.H. 1931. Einfluss der Tageslänge auf die Wachstumsphasen des Salats. Genetische Untersuchungen I. Die Gartenbauwissenschaft 4:469-483.

Carey, E.E., L. Jett, W.J. Lamont, Jr., T.T. Nennich, M.D. Orzolek, and K.A. Williams. 2009. Horticultural crop production in high tunnels in the United States: A snapshot. HortTechnology 19:37-43.

Dufault, R.J., B. Ward, and R.L. Hassell. 2006. Planting date and romaine lettuce cultivar affect quality and productivity. HortScience 41:640-645.

Dufault, R.J., B. Ward, and R.L. Hassell. 2009. Dynamic relationships between field temperatures and romaine lettuce yield and head quality. Sci. Hort. 120:452-459.

Fehr, W.R. 1987. Principles of cultivars development: Theory and technique. Macmillan, New York, NY.

Fukuda, M., S. Matsuo, K. Kikuchi, W. Mitsuhashi, T. Toyomasu, and I. Honda. 2009. The endogenous level of $\mathrm{GA}_{1}$ is upregulated by high temperature during stem elongation in lettuce through LsGA3ox I expression. J. Plant Physiol. 66:2077-2084.

Fukuda, M., S. Matsuo, K. Kikuchi, W. Mitsuhashi, T. Toyomasu, and I. Honda. 2012. Gibberellin metabolism during stem elongation stimulated by high temperature in lettuce. Acta Hort. 932:359-364.

Harwood, T.D., F.A. Al Said, S. Pearsona, S.J. Houghton, and P. Hadley. 2010. Modelling uncertainty in field grown iceberg lettuce production for decision support. Comput. Electron. Agr. 71:57-63.

Jenni, S. and W. Yan. 2009. Genotype by environment interactions of heat stress disorder resistance in crisphead lettuce. Plant Breed. 128:374-380.

Kadir, S., E. Carey, and S. Ennahli. 2006. Influence of high tunnel and field conditions on strawberry growth and development. HortScience 41:329-335.

Kristensen, S., E. Friis, K. Henriksen, and S.A. Mikkelsen. 1987. Application of temperature sum in the timing of production of crisp lettuce. Acta Hort. 198:217-226.

Lafta, A. and B. Mou. 2013. Evaluation of lettuce genotypes for seed thermotolerance. HortScience 48:708-714.

Lamont, W.J., M. McGann, M. Orzolek, N. Mbugua, B. Dye, and D. Reese. 2002. Design and construction of the Penn State high tunnel. HortTechnology 12:447-453.

Lamont, W.J. 2005. Plastics: Modifying the microclimate for the production of vegetable crops. HortTechnology 15:477-481.

Lamont, W.J. 2009. Overview of the use of high tunnels worldwide. HortTechnology 19:25-29.

Lee, O.N., N. Sugiyama, and S. Kosuge. 2003. Allometry of stem growth in lettuce plants. J. Jpn. Soc. Hort. Sci. 72(1):24-28.

Montgomery, D.C. 1984. Multifactor experiments with randomization restrictions, p. 379-397. In: Design and analysis of experiments. Wiley, New York, NY.

Mou, B. 2005. Genetic variation of Beta-carotene and lutein contents in lettuce. J. Amer. Soc. Hort. Sci. 130:870-876.
Mou, B. 2008. Lettuce, p. 75-116. In: J. Prohens and F. Nuez (eds.). Handbook of plant breeding. Vegetables I. Asteraceae, Brassicaceae, Chenopodiaceae, and Cucurbitaceae. Springer Science, New York, NY.

Nagata, R.T. 1992. Clip-and-wash method of emasculation for lettuce. HortScience 27:907908.

Neter, J., W. Wasserman, and M.H. Kutner. 1989. Multiple linear regression, p. 223-270. In: Applied linear regression models. Irwin, Boston, MA.

Nothmann, J. 1977. Morphogenetic effects of seasonal conditions on head development of cos lettuce (Lactuca sativa cv. Romana) growing in subtropical climate. J. Hort. Sci. 52:155162.

Orzolek, M.D., W.J. Lamont, and L. White. 2004. Promising horticultural crops for production in high tunnels in the mid-Atlantic area of the United States. Acta Hort. 633:453-458.

Rader, H.B. and M.G. Karlsson. 2006. Northern field production of leaf and romaine lettuce using a high tunnel. HortTechnology 16:649654.

Rappaport, L. and S.H. Wittwer. 1956a. Flowering in head lettuce as influenced by seed vernalization, temperature, and photoperiod. Proc. Amer. Soc. Hort. Sci. 67:429-437.

Rappaport, L. and S.H. Wittwer. 1956b. Night temperature and photoperiod effects on flowering of leaf lettuce. Proc. Amer. Soc. Hort. Sci. 68:279-282.

Reeves, J. and D. Drost. 2012. Yields and soil quality under transitional organic high tunnel tomatoes. HortScience 47:38-44.

Reiss, E., A.J. Both, S. Garrison, W. Kline, and J. Sudal. 2004. Season extension for tomato production using high tunnels. Acta Hort. 659:153-160.

Thompson, H.C. and J.K. Knott. 1933. The effect of temperature and photoperiod on the growth of lettuce. Proc. Amer. Soc. Hort. Sci. 30:507509.

Wallace, R.W., A.L. Wszelaki, C.A. Miles, J.S Cowan, J. Martin, J. Roozen, B. Gundersen, and D.A. Inglis. 2012. Lettuce yield and quality when grown in high tunnel and open-field production systems under three diverse climates. HortTechnology 22:659-668.

Waterer, D. 2003. Yields and economics of high tunnels for production of warm-season vegetable crops. HortTechnology 13:339-343.

Waycott, W. 1995. Photoperiodic response of genetically diverse lettuce accessions. J. Amer. Soc. Hort. Sci. 120:460-467.

Wells, O.S. and J.B. Loy. 1993. Rowcovers and high tunnels enhance crop production in the northeastern United States. HortTechnology 3:92-95.

Wiebe, H.J. and H. King. 1985. Influence of daylength on development and growth of lettuce. Gartenbauwissenschaft 50:202-206.

Wur, D.C.E., J.R. Fellows, R.W.P. Hiron, D.N. Antill, and D.J. Hand. 1992. The development and evaluation of techniques to predict when to harvest iceberg lettuce heads. J. Hort. Sci. 67:385-393.

Zhao, X., E.E. Carey, and M. Hall. 2003. Influence of protected environment and fertilizer source on growth and quality of lettuce and leafy greens during summer production in Kansas. HortScience 38:749 (abstr.).

Zhao, X. and E.E. Carey. 2009. Summer production of lettuce, and microclimate in high tunnel and open field plots in Kansas. HortTechnology 19:113-119. 